\title{
Hybrid Fuzzy and Data-Driven Robust Optimization for Resilience and Sustainable Health Care Supply Chain with Vendor-Managed Inventory Approach
}

\author{
Reza Lotfi ${ }^{1,2}$ (D) Bahareh $\operatorname{Kargar}^{3} \cdot$ Mohsen Rajabzadeh $^{4}$ Fatemeh Hesabi $^{5}$ • \\ Eren Özceylan ${ }^{6}$
}

Received: 16 June 2021/Revised: 15 October 2021 / Accepted: 19 October 2021/Published online: 1 February 2022

(C) Taiwan Fuzzy Systems Association 2022

\begin{abstract}
One of the problems that government managers deal with are medical inventory management in COVID-19 conditions. Based on this situation, the best strategy for managing and reducing inventory costs can be VendorManaged Inventory (VMI) policy in the recent decade. Therefore, a hybrid fuzzy and data-driven robust optimization for Resilience and Sustainable Health Care Supply Chain (RSHCSC) with VMI approach is appropriate for improving the inventory management system and tackling uncertainty and disruption in this situation. Three RSHCSC models are suggested using hybrid fuzzy and data-driven robust optimization with a stochastic programming
\end{abstract}

Reza Lotfi

reza.lotfi.ieng@gmail.com; rezalotfi@stu.yazd.ac.ir

Bahareh Kargar

kargar.iust@gmail.com

Mohsen Rajabzadeh

M.rajabzadeh@modares.ac.ir

Fatemeh Hesabi

Fatemehhesabi@modares.ac.ir

Eren Özceylan

eozceylan@gantep.edu.tr

1 Department of Industrial Engineering, Yazd University, Yazd, Iran

2 Behineh Gostar Sanaye Arman, Tehran, Iran

3 School of Industrial Engineering, Iran University of Science and Technology, Tehran, Iran

4 Department of Business Administration, Kheradgarayan Motahar Institute of Higher Education, Mashhad, Iran

5 Department of Information Technology Management, Tarbiat Modares University, Tehran, Iran

6 Department of Industrial Engineering, Gaziantep University, Gaziantep, Turkey approach. The first model is average and mean absolute function, the second model is Conditional Value at Risk (CVaR), the third model is Minimax model, and the final model is the traditional inventory model. Each of the proposed models has advantages and disadvantages that depend on the conservative level of decision-maker. Sensitivity analysis is done on essential parameters like fuzzy cut, confidence level, robust and resilience coefficient, and size models. The results show that increasing fuzzy cut, confidence level, robustification coefficient, resiliency coefficient, and CVaR confidence level amount of costs grows. The Minimax function is suitable for conservative decision-makers.

Keywords Data-driven robust optimization - Fuzzy · Health care supply chain · Resilience - Sustainable . Vendor-managed inventory

\section{Introduction}

One of the best strategies that have been applied with companies for managing and reducing the cost of inventory is vendor-managed inventory (VMI) in the recent decade. The vendor manages and controls inventory along the supply chain (SC) in this policy. Many retailers used this strategy for their suppliers, such as Wal-Mart [1]. Using this strategy can help managers of health care SC to improve drug inventory agility [2]. This strategy enables organizations to share information between vendors (suppliers) and retailers like hospital drug stores. When information sharing is implemented correctly between vendors and retailers, vendors are aware of the inventory level in retailers [3-5]. 
Moreover, researchers suggested using blockchain to manage information sharing between SC components [6-8]. Also, having smart contract systems, like the Ethereum network, increases productivity in a chain and helps to run VMI efficiently [8]. Therefore, we need to establish VMI to increase resiliency and robustness in health care SC

The research challenges and research problems make the design resilient and robust to decrease risks in SC. Attention to resilience and robustness can increase SC flexibility to cope with demand fluctuation and natural occurrence disruption [9]. Designing a sustainable and green SC [10] with resilience and robust VMI considering risk can help stockholders manage costs, environmental, energy, and social impact [11]. We need to move to smart SC.

As a result, the innovation and contribution of this research is as follows:

1. Designing resilience and sustainable health care SC,

2. Appling VMI in health care SC,

3. Appling data-driven robust optimization in health care SC to cope and tackle uncertainty and disruption.

The paper is organized as follows. Section 2 survey of related work on application of VMI. In Sect. 3, the mathematical problem is stated. Section 4 presents the findings of research and sensitivity analysis in a case study. In Sect. 5, the managerial implications and practical insights are described. In Sect. 6, the conclusion is summarized.

\section{Survey of Related Work on VMI}

Since the concept of VMI defines until now, much research is presented in the VMI Area with different tools. Some researchers compared traditional inventory management with VMI and showed that using this policy helps stockholders, others developed in solution approach.

\subsection{Application of VMI in Green Supply Chain}

Fakhrzad and Lotfi [11] investigated VMI in a green SC with backorder. The authors developed paper of Diabat [12], added green objectives and backorder to his model, and compared with traditional inventory models. They employed $\varepsilon$-constraint, Non-Dominated Sorting Genetic Algorithm (NSGA-II) to solve the model on small scale and large scale, respectively. They found that establishing VMI increases the profit.

Gharaei et al. [13] studied green SC with VMI approach and with Consignment Stock (CS) agreement. Their model was single vendor and multi-buyer. The objective of the model was the total cost of SC with tax cost of emission, penalty cost, and financial constraints. They applied Outer
Approximation with Equality Relaxation and Augmented Penalty (OA/ER/AP).

Bai et al. [14] used VMI to manage deteriorating products and consider carbon emission for facilities. Their model consists of a single vendor and two competing retailers for deteriorating products. They compared the system with centralized, decentralized conditions and decentralized with revenue sharing. They found that profit and emission in a centralized SC is better than a decentralized SC.

To reduce unnecessary costs and $\mathrm{CO}_{2}$ emissions, Stellingwerf et al. [15] suggested eco-efficient VMI cooperation to control cost and environmental impact for transportation in SC. They applied Cooperative Game Theory (CGT) and Shapley value to calculate profit in supermarket chains in the Netherlands. Results showed that eco-efficient VMI cooperation obtained lower cost and emission than traditional inventory management.

Karampour et al. [16] studied a bi-objective green VMI problem. The objective of the model includes maximizing the profit and minimizing the carbon emissions of transportation. The model constraints were the maximum capacity of vendor to retailers, maximum number of received orders from retailers, and the capacity of retailers. They embedded NSGA-II, Multi-Objective of Keshtel Algorithm (MOKA), and Multi-Objective of Red Deer Algorithm (MORDA). They compared these algorithms with Epsilon-constraint ( $\varepsilon$-constraint). They found that MORDA has better performance than MOKA and NSGAII.

Jamshidpour Poshtahani and Pasandideh [17] suggested a green SC with a bi-objective VMI in a multi-product EPQ model and chance constraint for uncertainty. To consider environmental impact, they added Green House Gas (GHGs) emissions to the model. LP-metric, goal attainment, and multi-choice goal programming with utility function (MCGP-U) are applied to produce Pareto front.

\subsection{Application of VMI in Health Care Supply Chain}

By considering the importance of VMI in health care, Weraikat et al. [18] surveyed VMI in a pharmaceutical SC (PSC) and suggested a VMI system to communicate between producer and hospital to manage inventory of medicine. Adopting the VMI system for the PSC eliminated medication wastage. Also, information sharing of stock inventory with the producer led to better managing the medication replenishment and removing extra cost and shortage in PSC.

Also, Liu et al. [19] applied VMI routing in a blood SC (BLC). They decomposed the main problem to distribute and route planning sub-problem. An adaptive large 
neighborhood search (ALNS) algorithm is embedded to solve the model. The computation results showed that the VMI with routing could considerably reduce the operational cost of the BLC.

\subsection{Application of VMI in Other Situations of Supply Chain}

Sainathan and Groenevelt [20] surveyed coordination in SC by different inventory methods, i.e., Retailer Managed Inventory (RMI) and VMI. They used a scenario-based newsvendor problem with a single vendor and a single retailer. They studied five contracts that included quantity flexibility, buyback, sales rebate, quantity discount, and revenue sharing. They found that revenue sharing and buyback contracts coordinate under VMI.

Ganesh Kumar and Uthayakumar [21] investigated five policies in SC. Their policies included traditional geometric shipment, CS geometric shipment (CSGS), CSGS with delaying the last delivery, CSGS with $k$-delayed deliveries, and CSGS with $k$-delayed equal deliveries. To solve the model, they suggested a Genetic Algorithm (GA). The comparison results show that the proposed model gives the minimum cost than other policies.

De Giovanni [22] used Artificial Intelligence (AI) to improve SC performance with VMI policy. They suggested AI forecast accurately and more suitable for high supply and demand errors. They applied Nash equilibrium to show that using AI can be helpful for the profit of manufacture and retailer and increase emission.

Taleizadeh et al. [23] presented VMI policy with $(r$, $Q)$ and $(R, T)$ replenishment policies and allowing partial backordering. Their model consists of a single vendor and a multi-retailer (buyer). Solution methodology was deriving to obtain an optimal solution. Results showed that VMI policy with $(r, Q)$ and $(R, T)$ replenishment policies have advantages and disadvantages in different contexts.

Golpîra [24] developed Construction SC (CSC) network design with VMI strategy with a multi-project multi-resource multi-supplier. They implemented VMI to run an intelligent logistics system to integrate and coordinate in the whole CSC.

Karimian et al. [25] suggested a multi-product Economic Production Quantity (EPQ) with a VMI approach in a stochastic environment. Their model consists of a single vendor and a multi-retailer. Their objectives include minimizing the mean and variance of cost. To solve the model, they employed Geometric Programming (GP). Their case study is an Iranian furniture SC. They showed that after implementing the model, they gained $17.78 \%$ improvement in total cost.

Wei et al. [26] investigated a stochastic learning effect on RMI and VMI policy in SC. Their model surveyed a
RMI, VMI, and centralized scenario and derived the equilibrium decisions. They concluded that amount of holding cost change conditions from VMI to RMI scenario. Using the inventory carryover option in the RMI scenario is higher than in the VMI scenario.

As regard, we classify and survey VMI in Table 1. According to the research gap (cf. Table 1), we can see that application of VMI in Health care SC is limited. As a result, research innovation and contribution present a DataDriven Robust optimization (DDRO) for Resilience and Sustainable health care SC (RSHCSC) with VMI approach and considering risk-averse. This subject helps to cope with uncertainty and disruption and makes to design resilience and sustainable SC.

\section{Problem Statement}

According to the COVID-19 outbreak and results of disruptions, the health care industry needs to pay more attention for medicine inventory management. It is necessary to use a suitable policy to medicine inventory management like VMI that is proved in many research is the best policy for vendors and buyers (retailers). In this research, we model a SC include vendors (suppliers) that sell medicine to hospitals and hospital drugstores (Fig. 1). In our model, we consider sustainability constraints like environmental and energy consumption. Also, our model tries to resilience order disruption. We tried to use a hybrid uncertainty approach to cope with demand and other parameters inconsistency.

\subsection{Assumptions and Notation List}

- There is a multi-vendor and multi-buyer SC based on VMI approach,

- There are $|P|$ products,

- The planning horizon is infinite,

- Shortage of demand product is allowed, and

- Time of delivering order is immediate [11].

First, decision indices, parameters, and variables are defined as follows:

Indices

$i \quad$ Index of vendor (supplier) $i \in I=\{1,2, \ldots, i\}$,

$j \quad$ Index of buyer (hospital) $j \in J=\{1,2, \ldots, j\}$,

$p \quad$ Index of product (medicine)

$p \in P=\{1,2, \ldots, p\}$,

Index of scenario $s \in S=\{1,2, \ldots, s\}$, 


\section{Parameters}

$d_{j p s} \quad$ Demand at buyer $j$ for product $p$ under scenario $s$

$\mathrm{As}_{i p} \quad$ Ordering cost in vendor $i$ for product $p$,

$\operatorname{Ar}_{j p} \quad$ Ordering cost in buyer $j$ for product $p$,

$\pi_{i p} \quad$ Backorder time-independent cost in vendor $i$ for product $p$,

$\hat{\pi}_{i p} \quad$ Backorder cost in vendor $i$ for product $p$ per time unit,

$\operatorname{hr}_{j p} \quad$ Holding cost in buyer $j$ for product $p$,

$\mathrm{ff}_{p} \quad$ Space required for product $p$,

$\widetilde{F} \quad$ Total spaces for all products in every transport,

$M \quad$ Total number of orders for all products

$p p_{s} \quad$ Probably of scenario $s$,

$\lambda \quad$ Coefficient of Resiliency and disruption in order,

$e_{i} \quad \mathrm{CO}_{2}$ emission produced in vendor $i$,

ee $\quad \mathrm{CO}_{2}$ emission produced for each order,

$\widetilde{\mathrm{Em}}_{i s}$ Maximum $\mathrm{CO}_{2}$ emission in vendor $i$ under scenario $s$,

$\mathrm{en}_{i} \quad$ Consumed energy in vendor $i$,

en Consumed energy for each order,

$\widetilde{\mathrm{Eg}_{i s}} \quad$ Maximum energy in vendor $i$ under scenario $s$,

$o_{i} \quad$ Employment and occupation in vendor $i$,

oo Employment and occupation for each order,

$\widetilde{\mathrm{Ocu}}_{i s} \quad$ Minimum employment and occupation in vendor $i$ under scenario $s$,

$\mathrm{bb}$ The proportion of shortage to order,

$z_{\alpha} \quad$ Confidence level $\alpha$,

$\delta \quad \delta$-Cut in fuzzy number,

$c_{i p}, c_{j p}, c_{p}, c_{i}, c$ Data-driven robust coefficients

\section{Decision Variables}

$q_{i j p s} \quad$ Order quantity transported from vendor $i$ to buyer $j$ for product $p$ under scenario $s$,

$b_{i j p s} \quad$ Shortage allowed from vendor $i$ to buyer $j$ for product $p$ under scenario $s$,

$\operatorname{dd}_{i j p s} \quad$ Assigned demand buyer $j$ for vendor $i$ for product $p$ under scenario $s$,

$\Gamma_{s} \quad$ Total cost under scenario $s$,

$Z \quad$ Total cost (objective function)

\subsubsection{RSHCSC with VMI Approach (Model 1)}

This section suggests RSHCSC with a VMI approach [27]. Our model used resiliency coefficient in orders by applying percent disruption, scenario-based, and sustainability by embedding emission, energy, and occupation in a mathematical problem.

Model 1 RSHCSC with VMI approach

$\min Z_{\mathrm{VMI}}=\sum_{s} p_{s} \Gamma_{s}+z_{\alpha / 2} \sum_{s} p_{s}\left|\Gamma_{s}-\sum_{s} p_{s} \Gamma_{s}\right|$

subject to

$$
\begin{aligned}
\Gamma_{s}= & \sum_{i} \sum_{j} \sum_{p}\left(\frac{\left(A s_{i p}+A r_{j p}\right) d d_{i j p s}}{q_{i j p s}}+h r_{j p} \frac{\left(q_{i j p s}-b_{i j p s}\right)^{2}}{2 q_{i j p s}}\right. \\
& \left.+\frac{\hat{\pi}_{i p} b_{i j p s}}{2 q_{i j p s}}+\frac{\pi_{i p} b_{i j p s} d d_{i j p s}}{q_{i j p s}}\right), \quad \forall s .
\end{aligned}
$$

Constraints of total order volumes:

$f f_{p} q_{i j p s} \leq \tilde{F}, \quad \forall i, j, p, s$.

Constraints of resiliency in orders:

$\frac{\operatorname{dd}_{i j p s}}{q_{i j p s}} \leq \lambda M, \quad \forall i, j, p, s$.

Constraints of shortage:

$b_{i j p s} \leq \mathrm{bb} q_{i j p s}, \quad \forall i, j, p, s$.

Constraints of demand assignment:

$\operatorname{dd}_{i j p s}=\frac{d_{j p s}}{|I|}, \quad \forall i, j, p, s$.

Constraints of sustainability (environments and energy consumption):

$e_{i}+e e \sum_{j} \sum_{p} q_{i j p s} \leq E m_{i s}, \quad \forall i, s$,

$e n_{i}+e n \sum_{j} \sum_{p} q_{i j p s} \leq E g_{i s}, \quad \forall i, s$,

$o_{i}+o o \sum_{j} \sum_{p} q_{i j p s} \geq O c u_{i s}, \quad \forall i, s$.

Decision variables:

$q_{i j p s}, b_{i j p s} \geq 0, \quad \forall i, j, p, s$.

As can be seen, the mathematical model is stochastic programming with scenario disruption. The objective function (1) minimizes the average costs and coefficient of mean absolute deviation for all scenarios. Constraint (2) includes ordering cost vendor and buyer, which is the vendor's responsibility and holding cost buyer and shortage cost. Constraint (3) guarantees that total order volumes from vendor $i$ to buyer $j$ for product $p$ under scenario $s$ are less than total spaces. Constraint (4) defines that order quantity for vendor $i$ to buyer $j$ for product $p$ under scenario $s$ is less than the total number of orders with resiliency. Constraint (5) defines that shortage should be less than the proportion of 


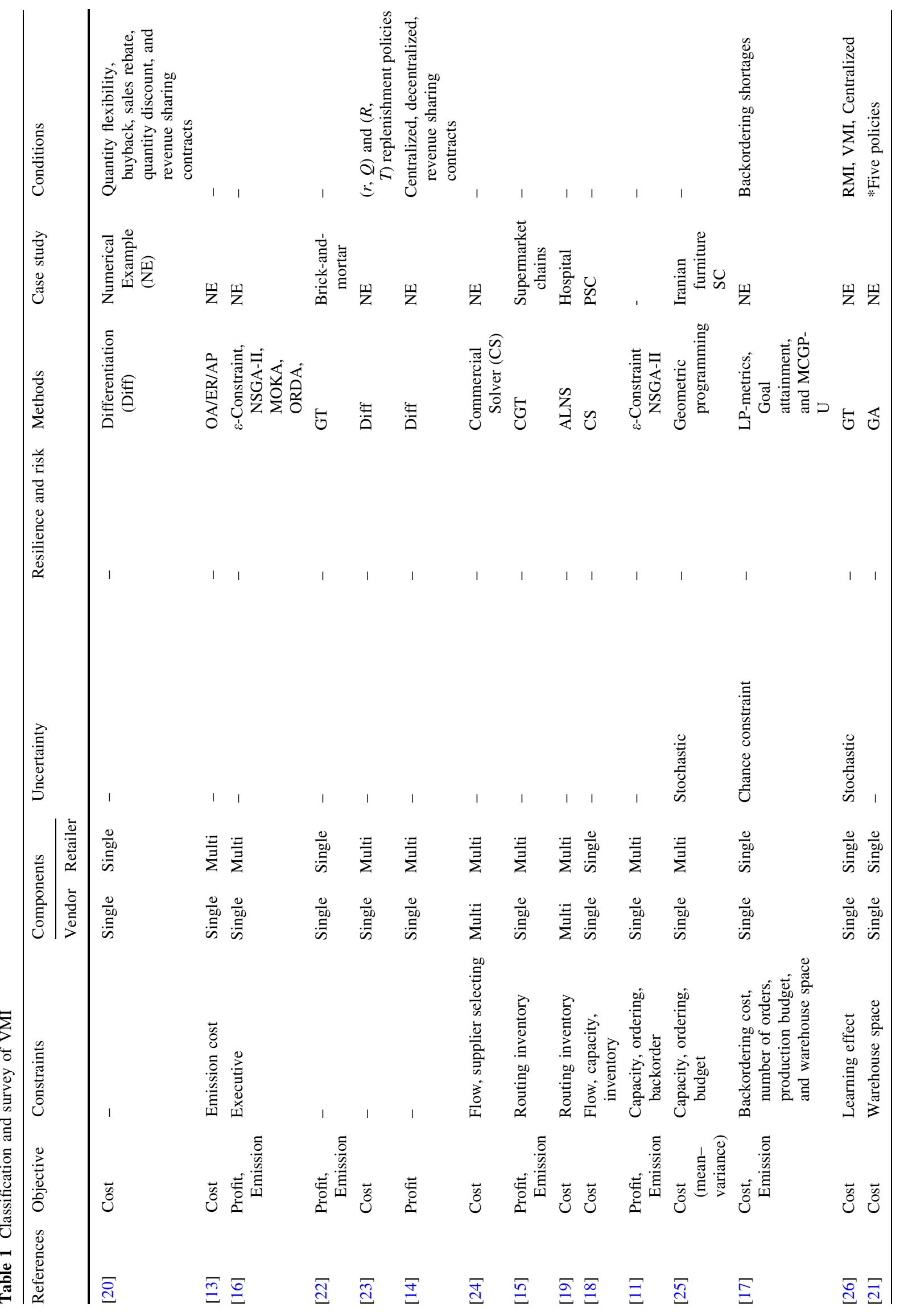




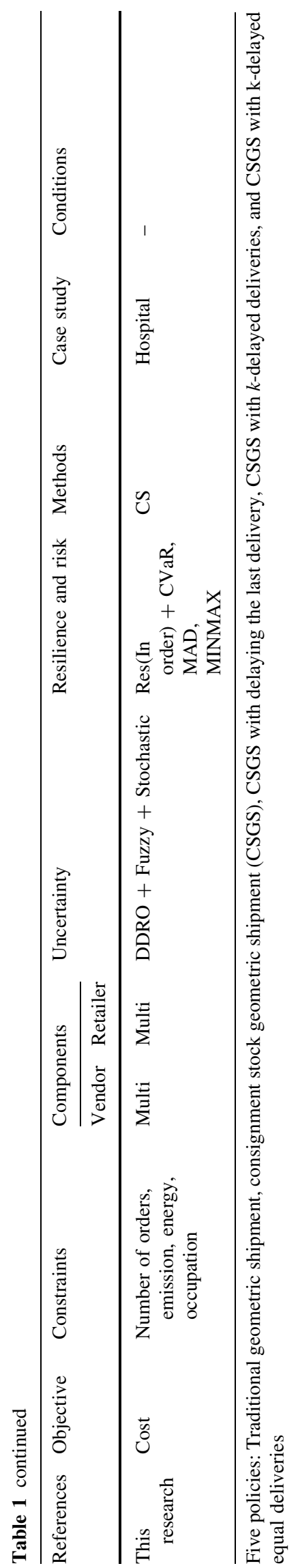




\section{Supplier (vendor) ( $i$ )}

\section{Hospital (buyer) (j)}

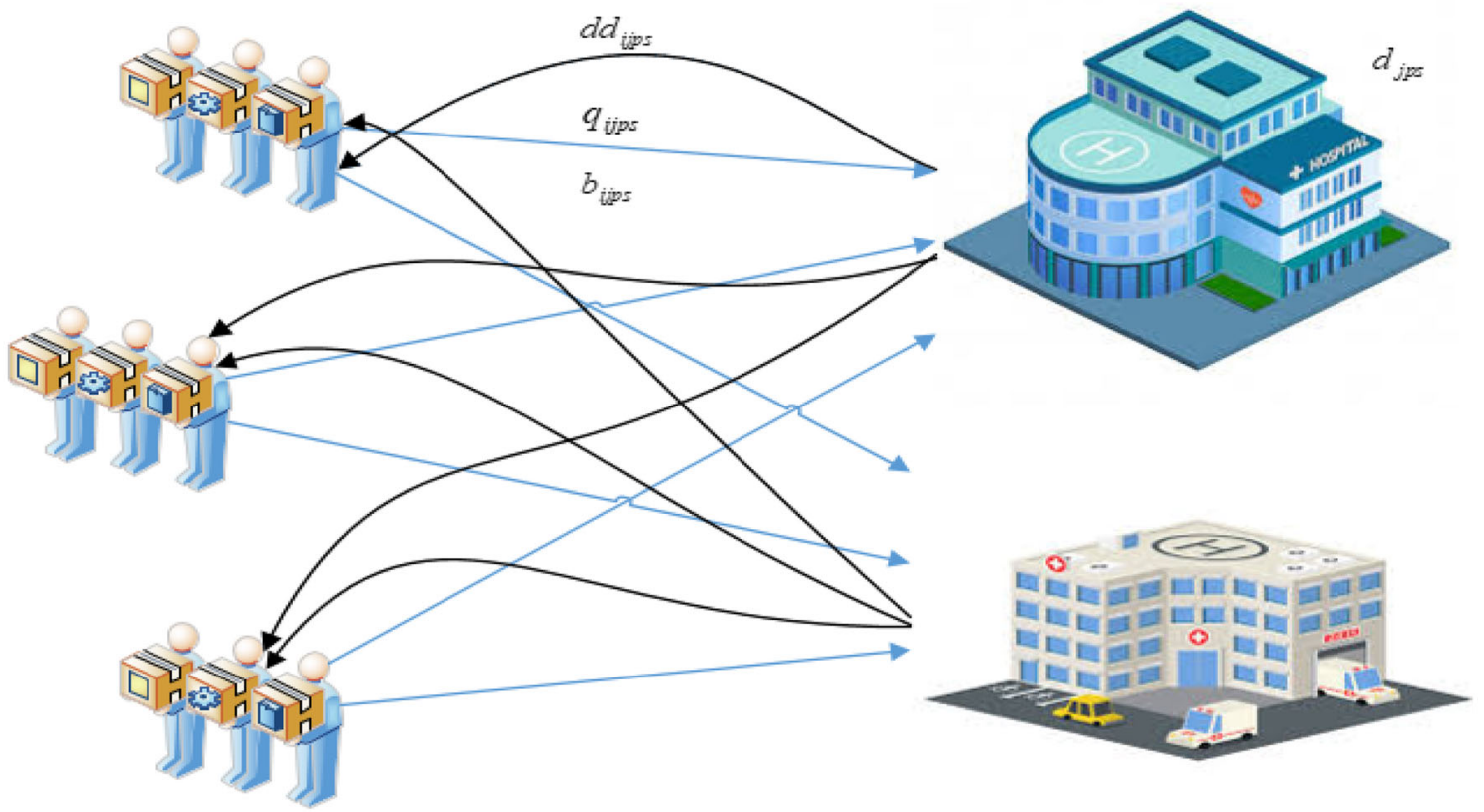

Fig. 1 Health care supply chain (medicine)

order. Constraint (6) presents the demand assignment to vendor $i$ from buyer $j$. Constraint (7) indicates that $\mathrm{CO}_{2}$ emission is less than allowed maximum emission. Constraint (8) indicates that consumed energy of SC should be less than allowed maximum energy. Constraint (9) expresses that amount of occupation is more than minimum occupation. Constraint (10) expresses positive decision variables.

\subsubsection{Linearization RSHCSC with VMI Approach (Model 1)}

Because of the absolute function in the objective function (1), we need to linearize the objective function and remove the absolute function. We replace absolute function with positive variables and add two constraints as follows [28]:

$\min Z_{\mathrm{VMI}}=\sum_{s} p_{s} \Gamma_{s}+z_{\alpha / 2} \sum_{s} p_{s}\left(v a_{s}+v b_{s}\right)$

subject to

$v a_{s}-v b_{s}=\Gamma_{s}-\sum_{s} p_{s} \Gamma_{s} \quad \forall s$,

$v a_{s}, v b_{s} \geq 0 \quad \forall s$.

Constraints (2)-(10).

\subsubsection{Defuzzification Method (Preliminary)}

As you can consider, if we have linear programming with fuzzy parameters, we can change to linear programming by Jiménez et al. [29] method: $\max Z=\sum_{j} c_{j} x_{j}$

subject to

$\sum_{j} a_{i j} x_{j} \leq \widetilde{b}_{i}, \quad \widetilde{b}_{i} \in\left(b_{i}^{1}, b_{i}^{2}, b_{i}^{3}\right) \quad \forall i$,

$\max Z=\sum_{j} c_{j} x_{j}$

subject to

$\sum_{j} a_{i j} x_{j} \leq \delta\left(\frac{b_{i}^{1}+b_{i}^{2}}{2}\right)+(1-\delta)\left(\frac{b_{i}^{2}+b_{i}^{3}}{2}\right), \quad \forall i$.

\subsubsection{Defuzzification RSHCSC with VMI Approach (Model 1)}

As you can consider, we have the fuzzy number and uncertainty parameters in this model. Some parameters have ambiguity like Total spaces $(\tilde{F})$, Maximum emission $\left(\widetilde{\mathrm{Em}}_{i s}\right)$, Maximum energy $\left(\widetilde{\mathrm{Eg}_{i s}}\right)$, and Minimum occupation $\left(\widetilde{\mathrm{Ocu}}_{i s}\right)$. Therefore, we need linear programming with fuzzy parameters $[29,30]$ :

We change Model (1) as follows:

$\min Z_{\mathrm{VMI}}=\sum_{s} p_{s} \Gamma_{s}+z_{\alpha / 2} \sum_{s} p_{s}\left(v a_{s}+v b_{s}\right)$

subject to 
$f f_{p} q_{i j p s} \leq \delta\left(\frac{F^{1}+F^{2}}{2}\right)+(1-\delta)\left(\frac{F^{2}+F^{3}}{2}\right)$,

$\forall i, j, p, s$,

$e_{i}+e e \sum_{j} \sum_{p} q_{i j p s} \leq \delta\left(\frac{\mathrm{Em}_{i s}^{1}+\mathrm{Em}_{i s}^{2}}{2}\right)+(1$

$-\delta)\left(\frac{\mathrm{Em}_{i s}^{2}+\mathrm{Em}_{i s}^{3}}{2}\right)$,

$\forall i, s$,

$e n_{i}+e n \sum_{j} \sum_{p} q_{i j p s} \leq \delta\left(\frac{\mathrm{Eg}_{i s}^{1}+\mathrm{Eg}_{i s}^{2}}{2}\right)+(1$

$-\delta)\left(\frac{\mathrm{Eg}_{i s}^{2}+\mathrm{Eg}_{i s}^{3}}{2}\right)$,

$\forall i, s$,

$o_{i}+o o \sum_{j} \sum_{p} q_{i j p s} \geq(1-\delta)\left(\frac{O c u_{i s}^{1}+O c u_{i s}^{2}}{2}\right)$

$+\delta\left(\frac{O c u_{i s}^{2}+O c u_{i s}^{3}}{2}\right)$,

$\forall i, s$.

Constraints (2)-(10), (12)-(13).

\subsubsection{Data-Driven Robust Approach (Preliminary)}

After that, we need to change uncertainty parameters to certainty parameters by Zhang et al. [31] method. They introduce a new data-driven robust optimization approach for a flexible box uncertainty set. Zhang et al. [31] defined the driven-robust optimization approach in Fig. 2.

You can see that a data-driven approach changes sharp box uncertainty to flexible box uncertainty. This method reduces the sharpness and makes it softer and smoother [32]. Therefore, it decreases conservative and risk-averse.

Suppose that linear programming with uncertainty parameters:

$\max Z=c x$

subject to

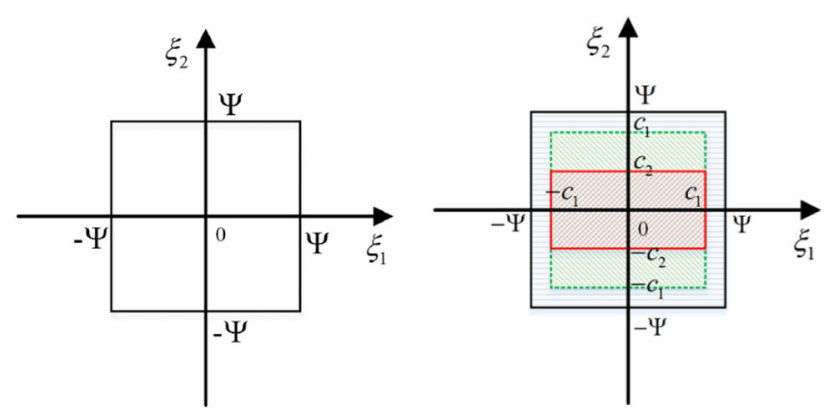

Fig. 2 A geometric view of classical and flexible box uncertainty [28]
$A x \leq \widehat{b} \quad(A, \widehat{b}) \in U$,

$x \in X$.

Above $(A, \hat{b})$ are the uncertainty matrix and be a member of the uncertainty flexible box set $J_{i}$. We assume

$\hat{a}_{i j}=a_{i j}+c_{i j} a_{i j} \quad \forall i, j \in J_{i}$,

$\widehat{b}_{i}=b_{i}+c_{i 0} b_{i} \quad \forall i, j \in J_{i}$,

$-1 \leq c_{i j}, \quad c_{i 0} \leq 1 \quad \forall i, j \in J_{i}$

where it is changed to

$\max Z=\sum_{j} c_{j} x_{j}$

subject to

$\sum_{j} a_{i j} x_{j}+\sum_{j \in J_{i}} c_{i j} \widehat{a}_{i j}\left|x_{j}\right|+c_{i 0} \widehat{b}_{i} \leq b_{i} \quad \forall i$.

Constraint (A6).

If $x_{j} \geq 0$ and all $j$ are member of $J_{i}$, we can write

$\max Z=\sum_{j} c_{j} x_{j}$

subject to

$\sum_{j}\left(a_{i j}+c_{i j} \widehat{a}_{i j}\right) x_{j}+c_{i 0} \widehat{b}_{i} \leq b_{i} \quad \forall i$

$x \geq 0$.

\subsubsection{Data-Driven Robust Approach for RSHCSC with VMI Approach (Model (1))}

Some parameters have deep uncertainty. Produced emission, energy consumption, and amount of employment have deep uncertainty in the vendor. Based on Sect. 3.2.4, we change Model (1) as follows:

$\min Z_{\mathrm{VMI}}=\sum_{s} p_{s} \Gamma_{s}+z_{\alpha / 2} \sum_{s} p_{s}\left(v a_{s}+v b_{s}\right)$

subject to

$$
\begin{aligned}
\Gamma_{s}= & \sum_{i} \sum_{j} \sum_{p}\left(\frac{\left(\mathrm{As}_{i p}+c_{i p} \widehat{\mathrm{As}}_{i p}+\mathrm{Ar}_{j p}+c_{j p} \widehat{\mathrm{Ar}}_{j p}\right) \mathrm{dd}_{i j p s}}{q_{i j p s}}\right. \\
& +\left(\mathrm{hr}_{j p}+c_{j p} \widehat{\mathrm{hr}}_{j p}\right) \frac{\left(q_{i j p s}-b_{i j p s}\right)^{2}}{2 q_{i j p s}} \\
& \left.+\frac{\left(\hat{\pi}_{i p}+c_{i p} \widehat{\hat{\pi}}_{i p}\right) b_{i j p s}}{2 q_{i j p s}}+\frac{\left(\pi_{i p}+c_{i p} \widehat{\pi}_{i p}\right) b_{i j p s} \mathrm{dd}_{i j p s}}{q_{i j p s}}\right), \quad \forall s,
\end{aligned}
$$




$$
\left(\mathrm{ff}_{p}+c_{p} \widehat{\mathrm{ff}}_{p}\right) q_{i j p s} \leq \delta\left(\frac{F^{1}+F^{2}}{2}\right)+(1-\delta)\left(\frac{F^{2}+F^{3}}{2}\right),
$$

$\forall i, j, p, s$,

$$
\begin{aligned}
& \left(e_{i}+c_{i} \widehat{e}_{i}\right)+(\mathrm{ee}+c \widehat{\mathrm{ee}}) \sum_{j} \sum_{p} q_{i j p s} \leq \delta\left(\frac{\mathrm{Em}_{i s}^{1}+\mathrm{Em}_{i s}^{2}}{2}\right) \\
& \quad+(1-\delta)\left(\frac{\mathrm{Em}_{i s}^{2}+\mathrm{Em}_{i s}^{3}}{2}\right), \\
& \quad \forall i, s,
\end{aligned}
$$

$$
\begin{aligned}
& \left(\mathrm{en}_{i}+c_{i} \widehat{\mathrm{en}}_{i}\right)+(\mathrm{en}+c \widehat{\mathrm{en}}) \sum_{j} \sum_{p} q_{i j p s} \leq \delta\left(\frac{\mathrm{Eg}_{i s}^{1}+\mathrm{Eg}_{i s}^{2}}{2}\right) \\
& \quad+(1-\delta)\left(\frac{\mathrm{Eg}_{i s}^{2}+\mathrm{Eg}_{i s}^{3}}{2}\right) \\
& \quad \forall i, s
\end{aligned}
$$

$$
\begin{aligned}
& \left(o_{i}+c_{i} \widehat{o}_{i}\right)+(\mathrm{oo}+c \widehat{\mathrm{oO}}) \sum_{j} \sum_{p} q_{i j p s} \geq(1 \\
& -\delta)\left(\frac{\mathrm{Ocu}_{i s}^{1}+\mathrm{Ocu}_{i s}^{2}}{2}\right)+\delta\left(\frac{\mathrm{Ocu}_{i s}^{2}+\mathrm{Ocu}_{i s}^{3}}{2}\right),
\end{aligned}
$$

$\forall i, s$.

\subsection{RSHCSC with VMI Approach and Considering Risk (Model (2))}

In this section, for comparing Model (1), we suggest RSHCSC with VMI approach and considering risk. We change objective (1) to CVaR [33]. CVaR is a coherent risk measure and computes expected shortfall at $\alpha$-level.

Model 2 RSHCSC with VMI approach and considering risk.

$\min Z_{\mathrm{VMI}}^{\prime}=\mathrm{CVaR}\left(\Gamma_{s}\right)$

subject to

$\operatorname{CVaR}\left(\Gamma_{s}\right)=\eta+\frac{1}{1-\alpha} \sum_{s} p_{s} v v_{s}, \quad \forall s$,

$v v_{s} \geq \Gamma_{s}-\eta \quad \forall s$.

Constraints (12)-(13), (19)-(23).

\subsection{RSHCSC with VMI and Worst-Case Approach (Model (3))}

Another method to compare Models (1) and (2) is using the worst-case method. We change objective (1) to the Minimax method. In this method, we minimize the maximum possible worst-case scenario.

Model 3 RSHCSC with VMI and worst-case approach. $\min Z_{\mathrm{VMI}}^{\prime \prime}=\operatorname{Max} \Gamma_{s}$ subject to

Constraints (12)-(13), (19)-(23).

Because of the max function in the objective function (26), we need to linearize the objective function and remove the max function. We replace a variable instead of the max function and add a constraint as follows:

$\min Z_{\mathrm{VMI}}^{\prime \prime}=\Delta$

subject to

$\Delta \geq \Gamma_{s} \quad \forall s$.

Constraints (12)-(13), (19)-(23).

\subsection{RSHCSC with No-VMI (Model (4))}

In this section, we compare main model with a situation without VMI. In this situation, the vendor and retailer are responsible for its inventory and ordering [27].

$$
\begin{aligned}
Z_{\text {NoVMI }}= & Z_{\text {NoVMI-Vendor }}\left(q_{i j p s}^{\text {Buyer }}\right) \\
& +\min \left(Z_{\text {NoVMI-Buyer }}\left(q_{i j p s}^{\text {Buyer }}\right)\right),
\end{aligned}
$$

$Z_{\text {NoVMI-Vendor }}\left(q_{i j p s}\right)=\sum_{s} \sum_{i} \sum_{j} \sum_{p} \frac{\left(\mathrm{As}_{i p}+c_{i p} \widehat{A s}_{i p}\right) \operatorname{dd}_{i j p s}}{q_{i j p s}}$,

$$
\begin{aligned}
Z_{\text {NoVMI-Buyer }}\left(q_{i j p s}^{\text {Buyer }}\right)= & \sum_{s} p_{s} \Gamma_{s} \\
& +Z_{\alpha / 2} \sum_{s} p_{s}\left|\Gamma_{s}-\sum_{s} p_{s} \Gamma_{s}\right|
\end{aligned}
$$

subject to

$$
\begin{aligned}
\Gamma_{s}= & \sum_{i} \sum_{j} \sum_{p}\left(\frac{\left(\operatorname{Ar}_{j p}+c_{j p} \widehat{\operatorname{Ar}}_{j p}\right) \mathrm{dd}_{i j p s}}{q_{i j p s}}+\left(\mathrm{hr}_{j p}+c_{j p} \widehat{\widehat{h}}_{j p}\right) \frac{\left(q_{i j p s}-b_{i j p s}\right)^{2}}{2 q_{i j p s}}\right. \\
& \left.+\frac{\left(\hat{\pi}_{i p}+c_{i p} \widehat{\hat{\pi}}_{i p}\right) b_{i j p s}}{2 q_{i j p s}}+\frac{\left(\pi_{i p}+c_{i p} \widehat{\pi}_{i p}\right) b_{i j p s} \mathrm{dd}_{i j p s}}{q_{i j p s}}\right), \quad \forall s .
\end{aligned}
$$

Constraints (12)-(13), (20)-(23).

\section{Numerical Analysis}

In this section, we set values for the parameters. Parameters include data for vendors that supply medicine for hospitals. Because our models are non-linear programming (NLP), we applied GAMS 24.1.2 software with BONMIN Solver for mathematical models with Processor Core i3-3210, CPU $3.2 \mathrm{GHz}, 8.00 \mathrm{~GB}$ RAM, operating system type 64-bit. We show the value of parameters for RSHCSC based on the notation list in Table 2 .

As you can see, variation of cost function (Model (1)) is based on confidence level ( $\alpha$-level) in Fig. 3 and Table 3. 
By increasing $\delta$-cut and increasing confidence level, amount of cost grows up. The reason is that space feasibility decreases and the cost grows up. It means that when the $\alpha$-level grows, the cost function is growing up.

\subsection{Variation Robustification Coefficient}

Also, the variation of cost function (Model (1)) based on robustification coefficient is shown in Fig. 4 and Table 4 . By increasing the robustification coefficient, the amount of costs grows up. It means that whatever robustification level coefficient is growing up, the cost function is growing up.

Variation of cost function (Model (3)) based on robustification coefficient is shown in Fig. 5 and Table 5. By increasing the robustification coefficient, the amount of cost grows up.

\subsection{Variation of Confidence Level of CVaR}

Variation of cost function (Model (2)) based on CVaR method is shown in Fig. 6 and Table 6. By increasing CVaR confidence level, amount of cost grows up. It means that whatever CVaR confidence level is growing up, the cost function is growing up.

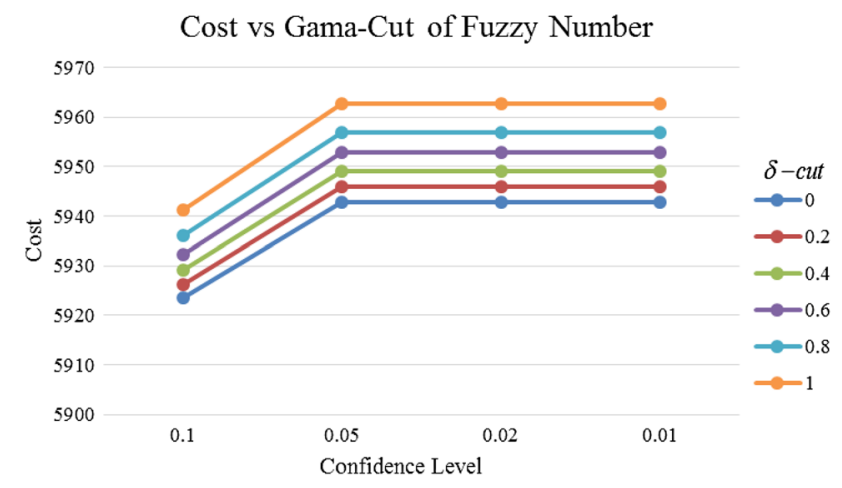

Fig. 3 Variation of cost function (Model (1)) $Z_{\mathrm{VMI}}$ based on $\alpha$-level

\subsection{Variation of Resiliency Coefficient}

Variation of cost function (Model (1)) based on resiliency coefficient is shown in Fig. 7 and Table 7. By decreasing the resiliency coefficient, amount of cost grows up. It means that when the resiliency coefficient decreased, cost function is growing up.

\subsection{Comparison of RSHCSC Models}

We compare three models that are proposed. Models (1) and (2) are overlapped. Model (3), which is the Minimax function, has the highest cost and is higher than Models (1)
Table 2 Table of parameters for RSHCSC

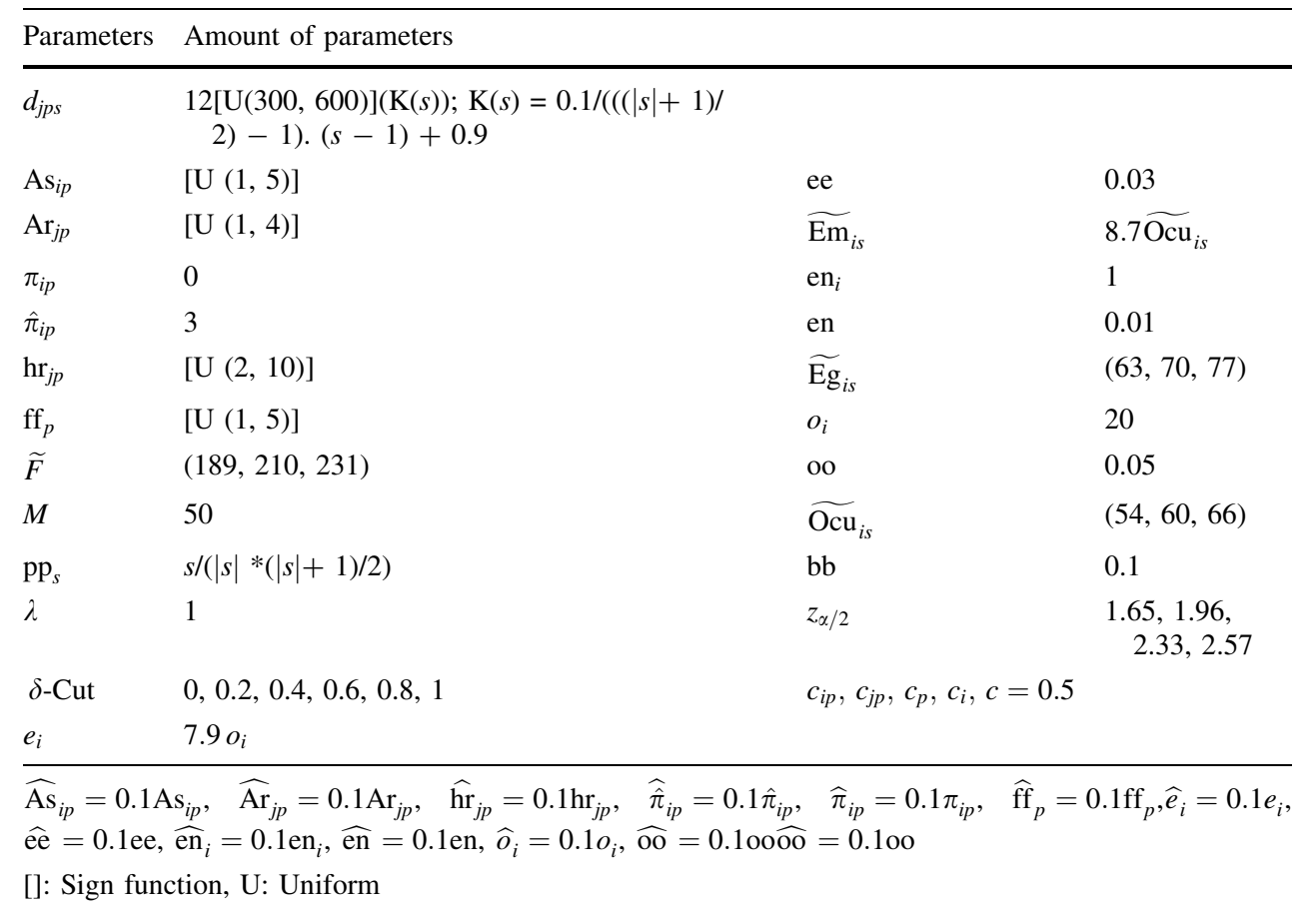


Table 3 Variation of cost function (Model (1)) $Z_{\mathrm{VMI}}$ based on $\alpha$-level

\begin{tabular}{llllll}
\hline$\delta$-Cut & $c$ & $1-\alpha=0.9$ & $1-\alpha=0.95$ & $1-\alpha=0.92$ & $1-\alpha=0.99$ \\
\hline 0 & 0.05 & 5923.534 & 5942.837 & 5942.837 & 5942.837 \\
0.2 & 0.05 & 5926.18 & 5945.842 & 5945.842 & 5945.842 \\
0.4 & 0.05 & 5929.124 & 5949.17 & 5949.17 & 5949.17 \\
0.6 & 0.05 & 5932.393 & 5952.841 & 5952.841 & 5952.841 \\
0.8 & 0.05 & 5936.009 & 5956.877 & 5956.877 & 5956.877 \\
1 & 0.05 & 5941.331 & 5962.672 & 5962.672 & 5962.672 \\
\hline
\end{tabular}

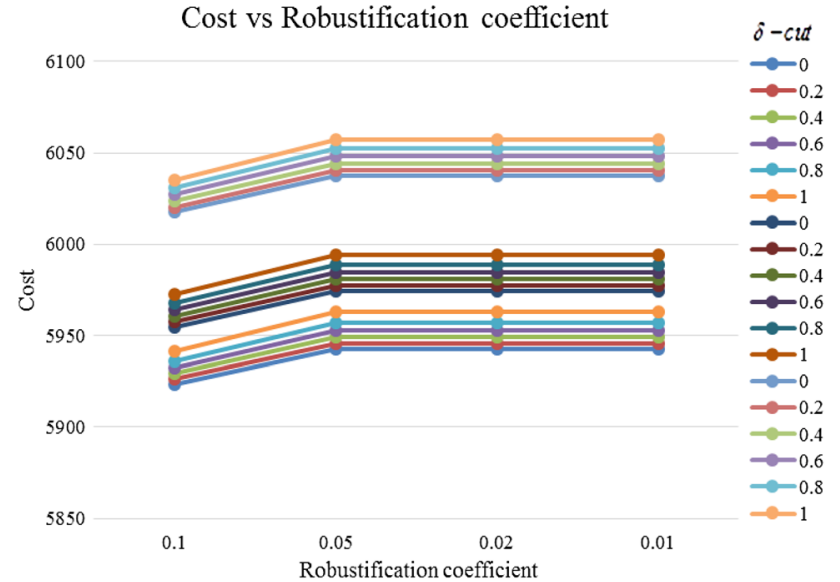

Fig. 4 Variation of cost function (Model 1) based on robustification coefficient

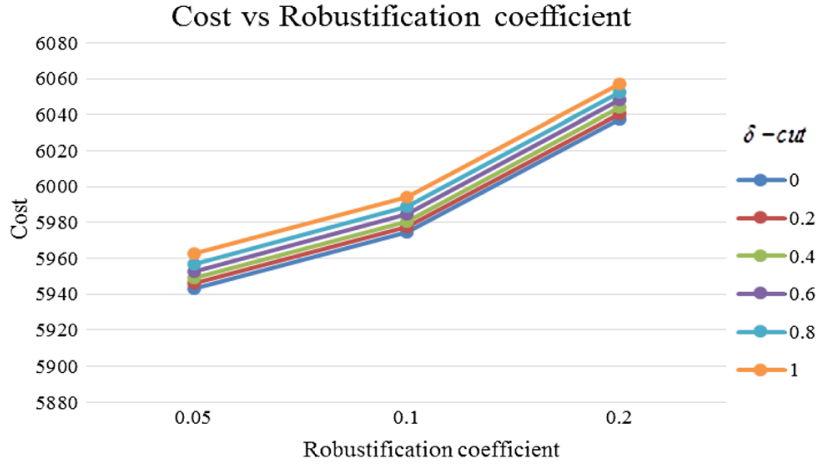

Fig. 5 Variation of cost function (Model (3)) $Z_{\mathrm{VMI}}^{\prime \prime}$ based on robustification coefficient
Table 4 Variation of cost function (Model (1)) $Z_{\mathrm{VMI}}$ based on robustification coefficient

\begin{tabular}{llllll}
\hline$\delta$-Cut & $c$ & $1-\alpha=0.9$ & $1-\alpha=0.95$ & $1-\alpha=0.92$ & $1-\alpha=0.99$ \\
\hline 0 & 0.05 & 5923.534 & 5942.837 & 5942.837 & 5942.837 \\
0.2 & 0.05 & 5926.18 & 5945.842 & 5945.842 & 5945.842 \\
0.4 & 0.05 & 5929.124 & 5949.17 & 5949.17 & 5949.17 \\
0.6 & 0.05 & 5932.393 & 5952.841 & 5952.841 & 5952.841 \\
0.8 & 0.05 & 5936.009 & 5956.877 & 5956.877 & 5956.877 \\
1 & 0.05 & 5941.331 & 5962.672 & 5962.672 & 5962.672 \\
0 & 0.1 & 5954.81 & 5974.299 & 5974.299 & 5974.299 \\
0.2 & 0.1 & 5957.528 & 5977.382 & 5977.382 & 5977.382 \\
0.4 & 0.1 & 5960.548 & 5980.792 & 5980.792 & 5980.792 \\
0.6 & 0.1 & 5963.898 & 5984.549 & 5984.549 & 5984.549 \\
0.8 & 0.1 & 5967.6 & 5988.674 & 5988.674 & 5988.674 \\
1 & 0.1 & 5972.502 & 5994.04 & 5994.04 & 5994.04 \\
0 & 0.2 & 6017.413 & 6037.28 & 6037.28 & 6037.28 \\
0.2 & 0.2 & 6020.277 & 6040.522 & 6040.522 & 6040.522 \\
0.4 & 0.2 & 6023.454 & 6044.097 & 6044.097 & 6044.097 \\
0.6 & 0.2 & 6026.968 & 6048.026 & 6048.026 & 6048.026 \\
0.8 & 0.2 & 6030.842 & 6052.33 & 6052.33 & 6052.33 \\
1 & 0.2 & 6035.282 & 6057.221 & 6057.221 & 6057.221 \\
\hline
\end{tabular}

and (2). If the decision-makers want to be conservative, they can use this form of objective and if he wants to be risk aware, he can use Models (1) and (2) (cf. Fig. 8; Table 8). Also, we compare three RSHCSC with VMI approach with the traditional model (No-VMI) and we can see that traditional model is higher cost than VMI model. Therefore, we recommend going to VMI for managers of health care SC. 
Table 5 Variation of cost function (Model (3)) $Z_{\mathrm{VMI}}^{\prime \prime}$ based on robustification coefficient

\begin{tabular}{llcl}
\hline$\delta$-Cut & \multicolumn{1}{l}{$c$} & \\
\hline & 0.05 & 0.1 & \multicolumn{1}{l}{0.2} \\
0 & 5942.837 & 5974.299 & 6037.28 \\
0.2 & 5945.842 & 5977.382 & 6040.522 \\
0.4 & 5949.17 & 5980.792 & 6044.097 \\
0.6 & 5952.841 & 5984.549 & 6048.026 \\
0.8 & 5956.877 & 5988.674 & 6052.33 \\
1 & 5962.672 & 5994.04 & 6057.221 \\
\hline
\end{tabular}

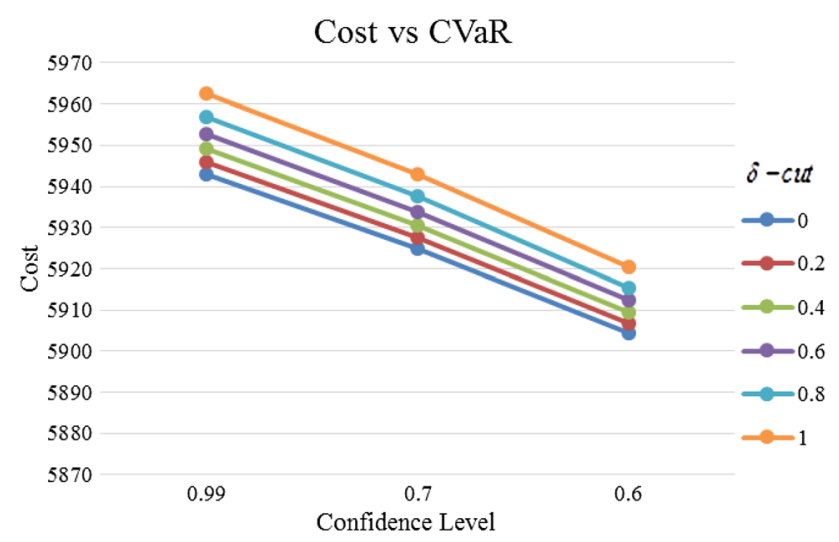

Fig. 6 Variation of cost function (Model (2)) $Z_{\mathrm{VMI}}^{\prime}$ based on CVaR

Table 6 Variation of cost function (Model (2)) $Z_{\mathrm{VMI}}^{\prime}$ based on CVaR

\begin{tabular}{|c|c|c|c|}
\hline \multirow[t]{2}{*}{$\delta$-Cut } & \multicolumn{3}{|l|}{$\operatorname{CVaR}(\alpha)$} \\
\hline & 0.99 & 0.7 & 0.6 \\
\hline 0 & 5942.837 & 5924.964 & 5904.537 \\
\hline 0.2 & 5945.842 & 5927.637 & 5906.831 \\
\hline 0.4 & 5949.17 & 5930.609 & 5909.397 \\
\hline 0.6 & 5952.841 & 5933.907 & 5912.269 \\
\hline 0.8 & 5956.877 & 5937.555 & 5915.473 \\
\hline 1 & 5962.672 & 5942.912 & 5920.329 \\
\hline
\end{tabular}

\subsection{The Complexity of RSHCSC Models}

Finally, we show free, positive variables and constraints for the complexity of the model in Eqs. (34)-(36). As you see, scenario sets size directly impacts time solution (Model (1)). By increasing scenario size, solution time grows exponentially (cf. Table 9; Figs. 9, 10). We need to use

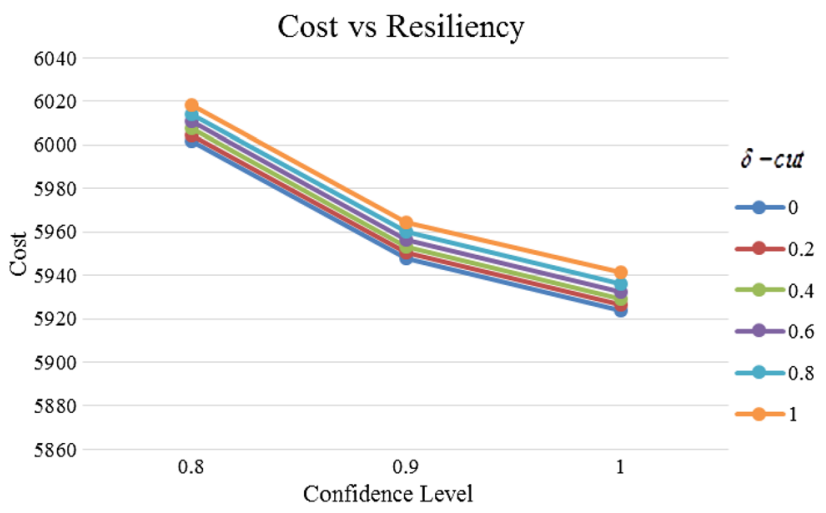

Fig. 7 Variation of cost function (Model (1)) $Z_{\mathrm{VMI}}$ based on resiliency coefficient

Table 7 Variation of cost function (Model (1)) $Z_{\mathrm{VMI}}$ based on resiliency coefficient

\begin{tabular}{llll}
\hline$\delta$-Cut & \multicolumn{3}{l}{ Resiliency $(\lambda)$} \\
\cline { 2 - 4 } & 0.8 & 0.9 & 1 \\
\hline 0 & 6001.774 & 5947.738 & 5923.534 \\
0.2 & 6004.421 & 5950.384 & 5926.18 \\
0.4 & 6007.364 & 5953.328 & 5929.124 \\
0.6 & 6010.633 & 5956.597 & 5932.393 \\
0.8 & 6014.25 & 5960.213 & 5936.009 \\
1 & 6018.24 & 5964.298 & 5941.331 \\
\hline
\end{tabular}

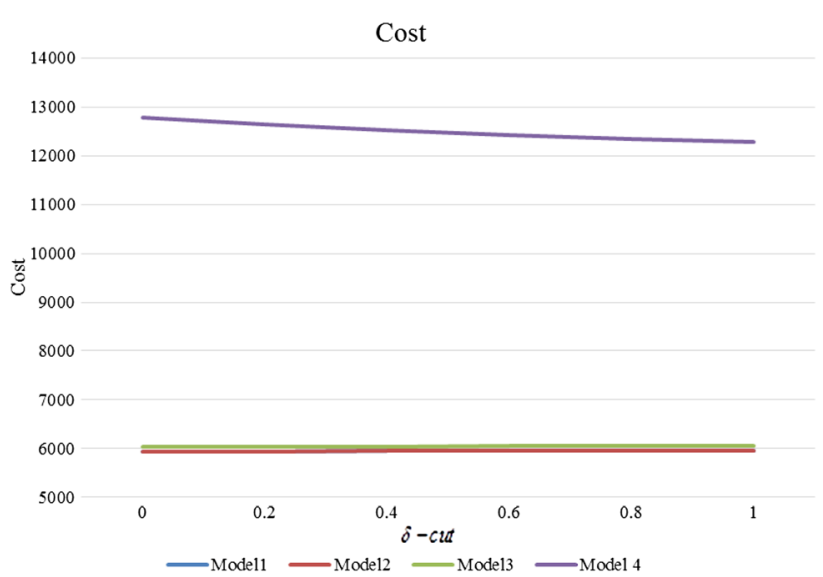

Fig. 8 Comparison models

heuristic and meta-heuristic to solve NLP model in minimum time [34, 35].

Free Variables $=4+|S|(|I||J||P|+1)$, 
Table 8 Comparison models

\begin{tabular}{lllll}
\hline$\delta$-Cut & Model $(1)-Z_{\mathrm{VMI}} 1-\alpha=0.99$ & Model $(2)-Z_{\mathrm{VMI}}^{\prime} 1-\alpha=0.99$ & Model (3)- $Z_{\mathrm{VMI}}^{\prime \prime}$ & Model $(4)-Z_{\mathrm{No}-\mathrm{VMI}} 1-\alpha=0.99$ \\
\hline 0 & 5942.837 & 5942.837 & 6037.28 & $12,791.495$ \\
0.2 & 5945.842 & 5945.842 & 6040.522 & $12,645.480$ \\
0.4 & 5949.17 & 5949.17 & 6044.097 & $12,524.057$ \\
0.6 & 5952.841 & 5952.841 & 6048.026 & $12,428.527$ \\
0.8 & 5956.877 & 5956.877 & 6052.33 & $12,345.182$ \\
1 & 5962.672 & 5962.672 & 6057.221 & $12,278.956$ \\
\hline
\end{tabular}

Table 9 Variation of cost function based on problem scale

\begin{tabular}{llllllr}
\hline Problems & $|I||J||P||S|$ & Free variables & Positive variables & Constraints & Cost function Model (1) & Times \\
\hline P1 & $2 * 2 * 5 * 5$ & 109 & 365 & 434 & 5941.331 & 2.402 \\
P2 & $5 * 5 * 5 * 5$ & 634 & 2015 & 2564 & $23,197.062$ & 10.961 \\
P3 & $10 * 10 * 6 * 6$ & 3610 & 11,178 & 14,536 & $88,590.013$ \\
P4 & $15 * 15 * 8 * 7$ & 12,611 & 38,661 & 50,628 & $213,458.5$ & 98.006 \\
P5 & $30 * 30 * 10 * 8$ & 72,012 & 218,424 & 288,500 & $764,930.54$ \\
P6 & $45 * 45 * 20 * 10$ & 405,014 & $1,224,030$ & $1,620,924$ & Not found \\
\hline
\end{tabular}

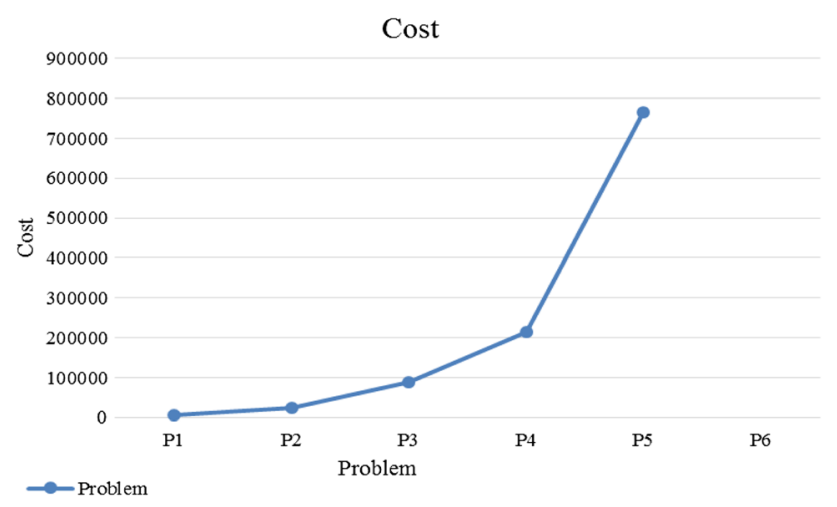

Fig. 9 Variation of cost function (Model (1)) based on problem scale

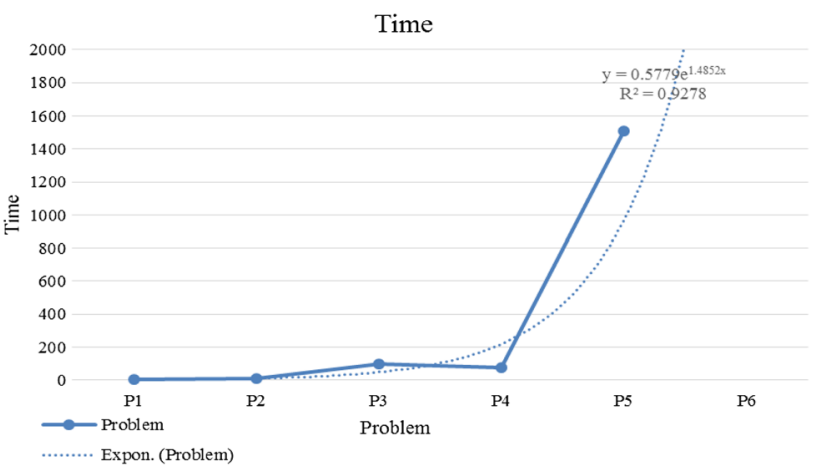

Fig. 10 Variation of cost function (Model (1)) based on the time solution

$$
\begin{aligned}
& \text { Positive Variables }=|S|(3|I||J||P|+|J||P|+3), \\
& \text { Constraints }=4+|S|(2+4|I||J||P|+2|I|) .
\end{aligned}
$$

\section{Managerial Implications and Practical Insights}

Many researches discussed about the advantages of VMI and this subject does not hide for any researchers and managers. In the COVID-19 situation, we should pay more attention to uncertainty in process design. As a result, we should use VMI in SC to decrease cost and environmental impact in this situation. We try to design resilient SC to tackle disruption and fluctuation of demand and other parameters by considering risk and uncertainty. We proposed a hybrid fuzzy and data-driven robust optimization for resilience and sustainable health care SC with a VMI approach. Three RSHCSC models with three conservative levels are proposed for designing a resilient, robust, and sustainable health care SC. Each model is suitable for kinds of conservative decision-makers. Risk-averse decisionmaker is better to use CVaR and Minimax model. The results show that applying VMI helps health care systems in hard situations like the COVID-19 pandemic to cope with uncertainty and resilience against demand fluctuation. Also, drug demand that needs patients is delivered as well. As managers of health care systems, we should embed this approach in SC to better performance and maximize competitive advantages. Therefore, we need a suitable model for each situation; it is better to select the CVaR 
and Minimax model, if we need to maximize the conservative level. Otherwise, we can select RSHCSC with VMI and hybrid fuzzy and data-driven.

\section{Conclusion}

Inventory management in the medical industry is the focus of managers of government in COVID-19 conditions. Based on these conditions, the best strategies that are needed to apply with companies in the recent decade for managing and reducing the cost of inventory can be VMI policy. Therefore, we show RSHCSC that used VMI strategy with considering risk-averse in health care SC. We used hybrid data-driven robust optimization, and fuzzy and stochastic programming approaches to cope with uncertainty to develop RSHCSC. This subject has not been surveyed by the researcher and can be innovative in this research. We suggested three kinds of objective functions. One of them includes average and MAD function costs; the second is the CVaR method and the third is the Minimax method.

The results of this research and managerial insights are as follows:

1. By increasing $\delta$-cut and increasing confidence level, amount of cost grows up (cf. Table 3; Fig. 3). By increasing the robustification coefficient, amount of cost grows up (cf. Fig. 4; Table 4). By decreasing the resiliency coefficient, amount of cost grows up (cf. Fig. 7; Table 7) (Model (1)).

2. Cost grows by the increase in the $\mathrm{CVaR}$ confidence level (cf. Fig. 6; Table 6) (Model (2)).

3. The cost increases by the increase in the robustification coefficient (cf. Fig. 5; Table 5) (Model (3)).

4. We compared three models and proposed Model (3) with a Minimax function and the highest cost. If the decision-maker wants to be conservative, he can use this form of objective and if he wants to be risk aware, he can use Models (1) and (2) (cf. Fig. 8; Table 8). Also, we compare all models with the traditional model (No-VMI) and we can see that the traditional model has a higher cost than the VMI model.

5. We show the complexity of Model (1). Scenario set sizes have a direct impact on time solution. By increasing scenario size, solution time grows up and goes to exponential time.

6. Scenario set size has a direct impact on the time solution. By increasing scenario size, solution time grows and goes to exponential time (cf. Figs. 9, 10; Table 9).

Regarding research constraints for solving models on a large scale, we suggest embedding heuristic and meta- heuristic algorithms to solve models for future research. Also, we recommend other coherent risk criteria like Entropic Value at Risk (EVaR) [36] and Robust Conditional Value at Risk (RCVaR) [37]. Using another method of data-driven robust optimization is an advantage for conservative decision-makers.

\section{References}

1. Dong, Y., Xu, K.: A supply chain model of vendor managed inventory. Transport. Res. E 38(2), 75-95 (2002)

2. Krichanchai, S., MacCarthy, B.L.: The adoption of vendor managed inventory for hospital pharmaceutical supply. Int. J. Logist. Manag. (2017). https://doi.org/10.1108/IJLM-01-20150010

3. Kaipia, R., Hartiala, H.: Information-sharing in supply chains: five proposals on how to proceed. Int. J. Logist. Manag. (2006). https://doi.org/10.1108/09574090610717536

4. Zaheer, N., Trkman, P.: An information sharing theory perspective on willingness to share information in supply chains. Int. J. Logist. Manag. (2017). https://doi.org/10.1108/IJLM-09-20150158

5. Ashraf, Z., et al.: Interval type-2 fuzzy vendor managed inventory system and its solution with particle swarm optimization. Int. J. Fuzzy Syst. 23, 1-26 (2021)

6. Guggenberger, T., Schweizer, A., Urbach, N.: Improving interorganizational information sharing for vendor managed inventory: toward a decentralized information hub using blockchain technology. IEEE Trans. Eng. Manag. 67(4), 1074-1085 (2020)

7. Casino, F., Dasaklis, T.K., Patsakis, C.: Enhanced vendor-managed inventory through blockchain. In: 2019 4th South-East Europe Design Automation, Computer Engineering, Computer Networks and Social Media Conference (SEEDA-CECNSM). IEEE (2019)

8. Omar, I.A., et al.: Enhancing vendor managed inventory supply chain operations using blockchain smart contracts. IEEE Access 8, 182704-182719 (2020)

9. Lotfi, R., et al.: A robust optimization model for sustainable and resilient closed-loop supply chain network design considering conditional value at risk. Numer. Algebra Control Optim. 11(2), 221-253 (2019)

10. Yu, Z., Khan, S.A.R.: Green supply chain network optimization under random and fuzzy environment. Int. J. Fuzzy Syst. (2021). https://doi.org/10.1007/S40815-020-00979-7

11. Fakhrzad, M.-B., Lotfi, R.: Green vendor managed inventory with backorder in two echelon supply chain with epsilon-constraint and NSGA-II approach. J. Ind. Eng. Res. Prod. Syst. 5(11), 193-209 (2018)

12. Diabat, A.: Hybrid algorithm for a vendor managed inventory system in a two-echelon supply chain. Eur. J. Oper. Res. 238(1), 114-121 (2014)

13. Gharaei, A., Karimi, M., Shekarabi, S.A.H.: An integrated multiproduct, multi-buyer supply chain under penalty, green, and quality control polices and a vendor managed inventory with consignment stock agreement: the outer approximation with equality relaxation and augmented penalty algorithm. Appl. Math. Model. 69, 223-254 (2019)

14. Bai, Q., Jin, M., Xu, X.: Effects of carbon emission reduction on supply chain coordination with vendor-managed deteriorating product inventory. Int. J. Prod. Econ. 208, 83-99 (2019) 
15. Stellingwerf, H., et al.: Fair gain allocation in eco-efficient vendor-managed inventory cooperation. J. Clean. Prod. 231, 746-755 (2019)

16. Karampour, M.M., et al.: Metaheuristics for a bi-objective green vendor managed inventory problem in a two-echelon supply chain network. Sci. Iran. (2020). https://doi.org/10.24200/sci. 2020.53420.3228

17. Jamshidpour Poshtahani, S., Pasandideh, S.H.R.: Optimizing a biobjective vendor-managed inventory of multi-product EPQ model for a green supply chain with stochastic constraints. J. Ind. Syst. Eng. 13(1), 1-34 (2020)

18. Weraikat, D., Zanjani, M.K., Lehoux, N.: Improving sustainability in a two-level pharmaceutical supply chain through Vendor-Managed Inventory system. Oper. Res. Health Care 21, 44-55 (2019)

19. Liu, W., et al.: Scheduling the distribution of blood products: a vendor-managed inventory routing approach. Transport. Res. E 140, 101964 (2020)

20. Sainathan, A., Groenevelt, H.: Vendor managed inventory contracts-coordinating the supply chain while looking from the vendor's perspective. Eur. J. Oper. Res. 272(1), 249-260 (2019)

21. Ganesh Kumar, M., Uthayakumar, R.: Modelling on vendormanaged inventory policies with equal and unequal shipments under GHG emission-trading scheme. Int. J. Prod. Res. 57(11), 3362-3381 (2019)

22. De Giovanni, P.: Smart Supply Chains with vendor managed inventory, coordination, and environmental performance. Eur. J. Oper. Res. 292(2), 515-531 (2020)

23. Taleizadeh, A.A., et al.: Stock replenishment policies for a vendor-managed inventory in a retailing system. J. Retail. Consum. Serv. 55, 102137 (2020)

24. Golpîra, H.: Optimal integration of the facility location problem into the multi-project multi-supplier multi-resource Construction Supply Chain network design under the vendor managed inventory strategy. Expert Syst. Appl. 139, 112841 (2020)

25. Karimian, Y., et al.: A geometric programming approach for a vendor managed inventory of a multiretailer multi-item EPQ model. RAIRO Oper. Res. 54(5), 1401-1418 (2020)

26. Wei, Q., et al.: Retailer vs. vendor managed inventory with considering stochastic learning effect. J. Oper. Res. Soc. 71(4), 628-646 (2020)

27. Pasandideh, S.H.R., Niaki, S.T.A., Nia, A.R.: An investigation of vendor-managed inventory application in supply chain: the EOQ model with shortage. Int. J. Adv. Manuf. Technol. 49(1-4), 329-339 (2010)

28. Hill, T.W., Ravindran, A.: On programming with absolute-value functions. J. Optim. Theory Appl. 17(1), 181-183 (1975)

29. Jiménez, M., et al.: Linear programming with fuzzy parameters: an interactive method resolution. Eur. J. Oper. Res. 177(3), 1599-1609 (2007)

30. Arana-Jimenez, M., Sánchez-Gil, M.C., Lozano, S.: Efficiencya assessment and target setting using a fully fuzzy DEA approach. Int. J. Fuzzy Syst. 22(4), 1056-1072 (2020)

31. Zhang, Y., Feng, Y., Rong, G.: New robust optimization approach induced by flexible uncertainty set: optimization under continuous uncertainty. Ind. Eng. Chem. Res. 56(1), 270-287 (2017)

32. Peng, W., et al.: Double-input rule modules stacked deep interval type-2 fuzzy model with application to time series forecasting. Int. J. Fuzzy Syst. 23, 1-21 (2021)

33. Zare Mehrjerdi, Y., Lotfi, R.: Development of a mathematical model for sustainable closed-loop supply chain with efficiency and resilience systematic framework. Int. J. Supply Oper. Manag. 6(4), 360-388 (2019)

34. Lotfi, R., et al.: Interdependent demand in the two-period newsvendor problem. J. Ind. Manag. Optim. 16(1), 117 (2020)
35. Lotfi, R., Amin Nayeri, M.: Multi-objective capacitated facility location with hybrid fuzzy Simplex and genetic algorithm approach. J. Ind. Eng. Res. Prod. Syst. 4(7), 81-91 (2016)

36. Ahmadi-Javid, A.: Entropic value-at-risk: a new coherent risk measure. J. Optim. Theory Appl. 155(3), 1105-1123 (2012)

37. Kara, G., Özmen, A., Weber, G.-W.: Stability advances in robust portfolio optimization under parallelepiped uncertainty. Cent. Eur. J. Oper. Res. 27(1), 241-261 (2019)

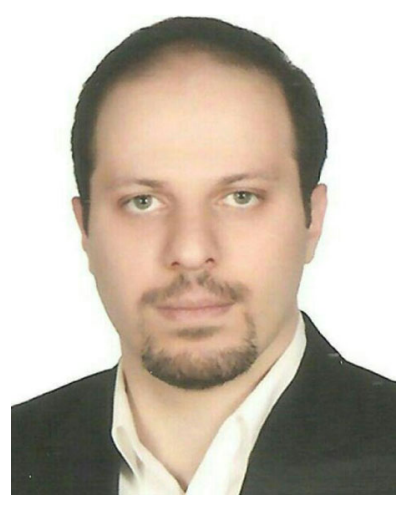

Reza Lotfi has done his Ph.D. Degree in Industrial Engineering from Yazd University (Iran, 2020). Currently, he is Director Manager of Behineh Gostar Sanaye Arman. He is Editor-inChief of the International Journal of Industrial engineering and Operational Research. He has published more than 14 papers in high-quality journals and conferences. He has been serving as a reviewer in many reputed journals such as Annals of Operations Research, Journal of Industrial and Management Optimization, and IEEE Transactions on Fuzzy Systems, Computational Intelligence, and Journal of Manufacturing Systems. He is an Excellent Reviewer in Computational Intelligence and Journal of Manufacturing Systems. His current research interests include supply chain management, robust optimization, inventory, project management, and renewable energy.

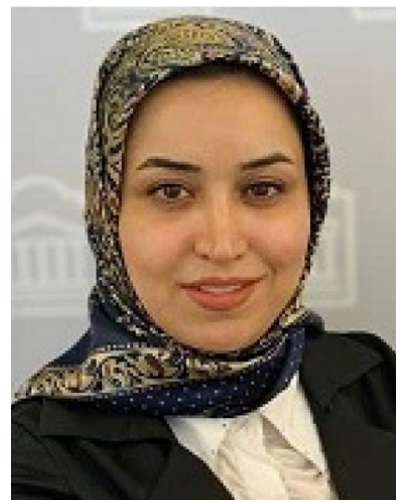

Bahareh Kargar is a $\mathrm{PhD}$ Student in Management at the University of Ottawa, specializing in Health Systems. Her research interests focus on operations research, health care management, decision-making under uncertainty, data analytics, and machine learning. Particularly, she is interested in the applications of machine learning and operations research in decision analysis and clinical decision support systems which help to improve decision-making in real-world problems. She holds a Master's Degree in Industrial Engineering from Iran University of Science and Technology. She has been a Teaching Assistant and Lab Instructor for Operations Management, Cost Accounting, and Managerial Accounting at the University of Ottawa. 


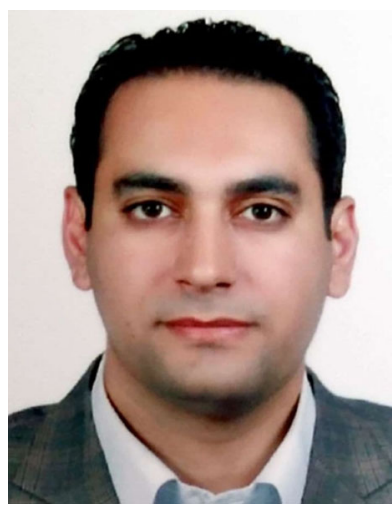

Mohsen Rajabzadeh is a Doctoral Researcher in the Department of Information Technology Management, Tarbiat Modares University, and Tehran, Iran. He is a Faculty Member in the Management Department, Kheradgarayan Motahar Institute of Higher Education, Mashhad, Iran. He has authored some papers and books in the area of e-government, knowledge management IoT, and supply chain. $\mathrm{He}$ is also an Excellent Reviewer in Quarterly Journal of Information Management Science and Technology and a Member of the Board of Directors of Noandishan Company.

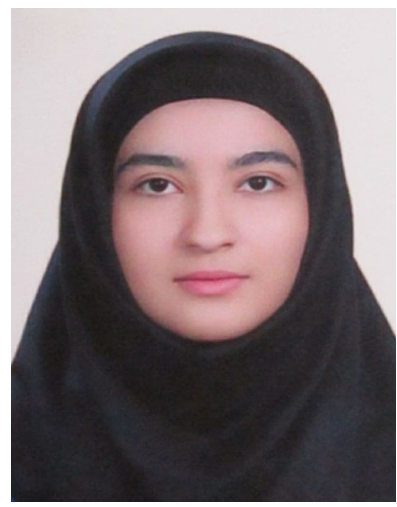

Fatemeh Hesabi is a Doctoral Researcher in the Department of Information Technology Management, Tarbiat Modares University, and Tehran, Iran. Her research interests center around the science and technology policy making, technology management, e-government, designing fuzzy expert systems, information technology policy making, and she has authored some papers and books in these areas.

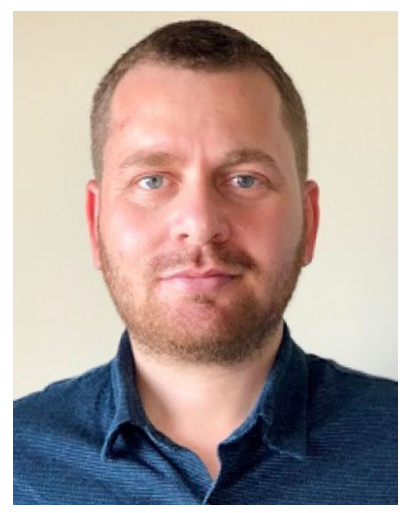

Eren Ozceylan is an Associate Professor in the Department of Industrial Engineering at the Gaziantep University, Turkey. He has earned B.S. and Masters in Industrial Engineering from Selçuk University, Turkey, and $\mathrm{Ph} . \mathrm{D}$. in Computer Engineering from Selçuk University. He was a Visiting Scholar at the Department of Mechanical and Industrial Engineering in Northeastern University, U.S.A. He has published more than 200 articles and conference papers in leading international journals and conferences. His research interests include environmental conscious production and distribution planning, GIS-based site selection, fuzzy mathematical programming, and disassembly line balancing. He is a Member of INFORMS and OR Society. 